\title{
Power spectra of uwb time-hopping modulated signals: a shot noise approach.
}

\author{
Pierre Bremaud ${ }^{\dagger \ddagger}$, Andrea Ridolfi ${ }^{\dagger 1}$ \\ $\dagger$ School of Computer and Communication Sciences, cole Polytechnique Fédérale de Lausanne, CH-1015 Lausanne. \\ $\ddagger$ INRIA-Ens, Dpartement d'Informatique, cole Normale Suprieure, 45 rue d'Ulm, F-75005 Paris.
}

pierre.bremaud@ens.fr, andrea.ridolfi@epfl.ch

\begin{abstract}
This paper presents a general method for obtaining the exact power spectra of generic time hopping modulated signals. Based on a point process approach, it provides simpler proofs for existing results and a powerful rigorous and at the same time systematic tool for computing the spectra of more complex time-hopping models. Spectrum formula are easy to understand and the contribution of each component of the model appears explicitly.
\end{abstract}

The family of pulse modulation techniques has gathered increasing interest since the introduction of ultra-wide band (UWB) impulse radio [1]. Exact spectral computation of pulse modulation techniques has already received the attention in the communication society $[1,4]$.

Here we focus on the power spectrum computation of the class of time hopping pulse position modulations [2], which is of particular interest for being well suited for multi access implementation. We exploit the fact that pulse modulated signals can be view as shot noises, i.e. as filtered point process. The corresponding models are simple and tractable and can be easily generalized to take into account other random quantities. Moreover, our approach can be extended toward a unifying model that also takes into account the family of pulse interval modulations [3]. The computation of the power spectral in then performed using recent results on the power spectrum of a shot noise $[5,6]$, leading to expressions of the spectra where the contribution of the various features of the model appears explicitly.

General pulse position time-hopping (тн) signals are modeled as follows [2]

$$
s(t)=\sum_{k=-\infty}^{\infty} A_{n} w\left(t-n T-B_{n} T_{b}-c_{n} T_{c}-\Theta_{n}\right)
$$

where

- $\left\{A_{n}\right\}$ and $\left\{B_{n}\right\}, n \in \mathbb{Z}$, are independent sequences of random variables that implement, respectively, pulse amplitude and pulse position modulation. $\left\{A_{n}\right\}, n \in \mathbb{Z}$ may also model pulse losses in the transmission;

- $\left\{c_{n}\right\}, n \in \mathbb{Z}$, is a deterministic periodic sequence, with period $L_{c}$, i.e. $c_{n}=c_{n+L_{c}}$.

- $\left\{\Theta_{n}\right\}, n \in \mathbb{Z}$, is a sequence of random variables, independent of $\left\{A_{n}\right\}$, and $\left\{B_{n}\right\}, n \in \mathbb{Z}$, that models the system clock jitter.

The time-hopping signal (1) is a shot noise with random excitation with filtering function given by $w\left(t-b T_{b}-\theta\right)$ and underlying point process $T_{n}=n T+c_{n} T_{c}+U=n L_{c} T+$

\footnotetext{
${ }^{1}$ Partially supported by the Swiss National Science Foundation under grant 21-65187.01.
}

$\sum_{l=0}^{L_{c}-1}\left(l T+c_{l} T_{c}\right)+U$ where $U$ is a $\left[0, L_{c} T\right]$-uniformly distributed random variable that guarantees the stationarity of the process. Then, the spectral "pseudo" density of a timehopping signal can be computed using the results on the spectrum of a shot noise with random excitation $[5,6]$, and is given by

$$
\begin{gathered}
f_{S}(\nu)=\frac{1}{L_{c} T}|\widehat{w}(\nu)|^{2} \\
\qquad \mathrm{E}\left[|A|^{2}\right] L_{c}\left\{1-\left|\varphi_{B}\left(2 \pi \nu T_{b}\right)\right|^{2}\left|\varphi_{\Theta}(2 \pi \nu)\right|^{2}\right\} \\
+\left|\varphi_{B}\left(2 \pi \nu T_{b}\right)\right|^{2}\left|\varphi_{\Theta}(2 \pi \nu)\right|^{2} \sum_{l, j=0}^{L_{c}-1} e^{2 i \pi \nu\left(\left(c_{l}-c_{j}\right) T_{c}+(l-j) T\right)} \\
\sum_{k \in \mathbb{Z}} C_{A}\left(k L_{c}+l-j\right) e^{2 i \pi \nu k L_{c} T} \\
+\left|\varphi_{B}\left(2 \pi \nu T_{b}\right)\right|^{2}\left|\varphi_{\Theta}(2 \pi \nu)\right|^{2} \sum_{l, j=0}^{L_{c}-1} e^{2 i \pi \nu\left(\left(c_{l}-c_{j}\right) T_{c}+(l-j) T\right)} \\
\left.|\mathrm{E}[A]|^{2} \frac{1}{L_{c} T} \sum_{n \neq 0} \delta\left(\nu-\frac{n}{L_{c} T}\right)\right\}
\end{gathered}
$$

where $\varphi_{Y}$ denotes the characteristic function of the random variable $Y$, and $C_{Y}$ its covariance.

The above formula is easily generalized to take into account additional random events: this allows for various extensions of published results.

\section{REFERENCES}

[1] M. Win and R. Scholtz, "Impulse radio: how it works," IEEE Communications Letters, vol. 2, no. 2, pp. 36-38, February 1998.

[2] M. Win and R. Scholtz, "Ultra-wide bandwidth time-hopping spread-spectrum impulse radio for wireless multiple-access communications," IEEE Trans. Comm., vol. 48, no. 4, pp. 679-689, April 2000.

[3] Z. Ghassemlooy, R. U. Reyher, E. D. Kaluarachchi, and A. J. Simmonds, "Digital pulse interval and width modulation," Microwave and Optical Technology Letter, vol. 11, no. 4, pp. 231236, 1996.

[4] M. Win, "A unified spectral analysis of generalized timehopping spread-spectrum signals in the presence of timing jitter," IEEE J. Select. Areas Commun., vol. 20, no. 9, pp. 16641676, December 2002.

[5] P. Brémaud, L. Massoulié, and A. Ridolfi, "Power spectra of random spike fields \& related processes," School of Computer and Communication Sciences, EPFL, Technical Report 200280, 2002, Submitted.

[6] P. Brémaud and A. Ridolfi, "Random spikes with correlated amplitudes: power spectrum and application to communications," School of Computer and Communication Sciences, EPFL, Tech. Rep., 2003. 